\title{
Author Correction: A strong and ductile medium-entropy alloy resists hydrogen embrittlement and corrosion
}

\author{
Hong Luo (D), Seok Su Sohn (D), Wenjun Lu, Linlin Li, Xiaogang Li (i), Chandrahaasan K. Soundararajan (D), \\ Waldemar Krieger, Zhiming Li (1) \& Dierk Raabe (D)
}

Correction to: Nature Communications https://doi.org/10.1038/s41467-020-16791-8, published online 17 June 2020.

The original version of this Article contained an error in the author affiliations.

The affiliation of Hong Luo and Xiaogang Li with Beijing Advanced Innovation Center for Materials Genome Engineering, Beijing 100083, China was inadvertently omitted.

This has now been corrected in both the PDF and HTML versions of the Article.

Published online: 02 July 2020

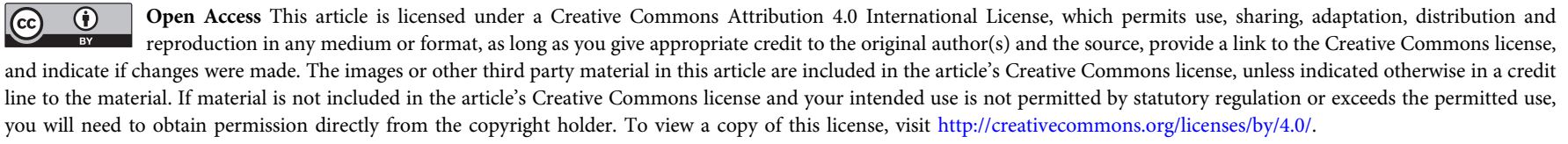

(C) The Author(s) 2020 